\title{
VANET Modelling and Statistical Properties of Connectivity in Urban Environment
}

\author{
Duong Hien Thuan ${ }^{1}$, Ho Van Cuu ${ }^{1}$, Hung Ngoc $\mathrm{Do}^{2}$ \\ ${ }^{1}$ Dept. of Electronics and Telecommunications, Sai Gon University, Vietnam \\ 2 School of Electrical Engineering, International University - Vietnam National University
}

Correspondence: Duong Hien Thuan, thuan.dh@cb.sgu.edu.vn

Manuscript communication: received 23 May 2013, accepted 17 July 2013

\begin{abstract}
This paper proposes a new approach for modelling Vehicular Ad Hoc Networks (VANETs) based on stochastic geometry and point processes, where road systems are modelled as random lines process and vehicle's locations on a road are linear Poisson point processes. We derive some functions, which show the connectivity properties of VANETs under the impact of fading and path loss, that can be used for large vehicular networks analysis.
\end{abstract}

Keywords- VANET, MANET, point process, Poisson point process, Poisson line process, stochastic geometry, V2V, V2R.

\section{INTRODUCTION}

In the last few years, advanced wireless technology and the breakthroughs in car industry made it possible to consider wireless Ad Hoc networks for providing connectivity (vehicle to vehicle $\mathrm{V} 2 \mathrm{~V}$ and vehicle to roadside V2R) among vehicles on the roads. Such network, referred to as VANET (Vehicular Ad Hoc Network), is seen to be one of the most valuable concept for improving road safety, transportation efficiency, and reduce the impact of transportation on environment. Because of the importance of these goals, several ongoing projects have been supported and involved various of members and organizations such as governments, academia, automotive industry, road operators, tolling agencies and other service providers [1]. Due to moving and random locations of vehicles and the effect of road system and fading channel, VANETs modelling is currently necessary demand to analyze, plan as well as design a network.

Most of researches were carried out by simulation, where results depend on specific simulation parameters setting. For example, reference [2] considered traffic flow metrics from the interaction of individual vehicles with each others in multi-lane high way scenario based on the Cellular Automata (CA) model. In [3], the authors modelled VANET in grid network with and without traffic light at an intersection and captured the connectivity probability from source node to arbitrary destination with the impact of market penetration, transmission range and roadside units by simulation and analysis based on percolation theory. The general mobility model with three phases and various speeds was presented in [4], where an open queuing theory was used to find spatial traffic distribution for vehicles in steady state of Sparse and Dense road segment with an impact of intersection. The authors in [5] introduced a hybrid simulator in macroscopic traffic model where statistical information propagation and communication protocol models were integrated. A model for VANETs simulating applied for city area was proposed in [6] with the changing in the speed of nodes after some specific distances was considered. CORNER [7] is a model that predicts path loss for simulation and shows the tradeoff between the accuracy of prediction and computational complexity. Some experimental works for collecting data about path loss and fading to build a statistical model for VANETs simulation was introduced in [8].

Although these simulation methods can show some characteristics of the VANETs for real applications, the main disadvantage of these is the difficulties in network analysis. Dealing with this problem, some VANET models were proposed for network performance analysis. In [9], the authors considered one-dimension multihop broadcast percolation with the impact of station density and transmission radius in which nodes were modelled as Poisson point process. Reference [10] derived probability that two nodes on an infinity line for a given distance can establish a multihop path between them in a simple deterministic channel by using percolation theory with Poisson boolean model. A street was modelled into discrete cells and the process of instantaneous information propagation to a given cell under uniform and general traffic streams was considered in [11]. In [12], the authors modelled one dimensional Ad Hoc network as $G / G / \infty$ by transforming connectivity distance distribution into the distribution of busy period of an equivalent infinite server queue. Another approach is modelled linear finite network by geometric graph (polytope graph) [13], in which authors investigated the impact of dynamic node pop- 
ulation and the delay constraint on connectivity. For the two-dimension networks, reference [14] shown the asymptotic critical transmission power (or range) for all nodes uniformly distributed in a disk-shaped network. In [15], the author expressed the critical transmitting range to ensure the network connected with nodes are uniformly distributed in region $[1, l]^{d}$ with $d=1,2,3$ ( $d$ is the number of dimension). The authors in [16] extended that problem by considering the presence of node mobility. The impact of log-normal shadowing on connectivity of Ad Hoc networks was first pointed out in [17] where nodes were modelled as Poisson point process. The similar issue was considered in [18] with geometric random graph model.

However, there are only a few researches investigated the effect of the channel randomness on VANET's performance evaluation. Almost researchers considered vehicular networks in high way scenario for one dimensional network model and Mobile Ad Hoc Networks (MANETs) in two dimensional network model in which the impact of road topology in urban environment on network performance metrics is ignored. The main contribution of this paper is to model the VANETs in urban areas scenario by stochastic geometry approach where the road system and vehicles's locations are taken into account by random processes.

The rest of this paper is organized as follows. In Section 2, we describe the stochastic geometry of road system, propagation channel model, point processes for vehicular's locations and a Euclidean distance distribution of the $n$th nearest neighbor. Section 3 analyzes some statistical properties of VANETs based on random point processes of proposed model. Numerical results of some examples of real DSRC (Dedicated Short Range Communication) system parameters are given in Section 4. Finally, the paper will be concluded in Section 5 .

\section{System Model Description}

\subsection{Road System Model}

The road systems can be modelled as random processes which depend on a few number of parameters and reproduce the statistical features of the real roads system such as length of road segments, number of crossings, total length of roads measured per unit area, number of quarters. In [19], roads system are presented as random geometric graph which are the edge sets of a random tessellation i.e. Poisson-Voronoi Tessellation (PVT), Poisson-Delaunay Tessellation (PDT), or Poisson Line Tessellation (PVT).

In this paper, a road is modelled as a random infinite straight line $G(p, \psi)$ as in figure 1 , where $p>0$ is a perpendicular distance from the line to the origin and $\psi \in[0,2 \pi]$ is an angle of the line with the abscissa axis, i.e., $x$ axis. We consider all roads have the same priority where there is no main road or side road. The mean length of the chord that a random line intercept in a bounded convex set $K$ with perimeter $L$ and area $F$ in a plane is given by $\mathbb{E}_{K}\{\sigma\}=\pi F / L$ [20, eq. (3.13)], where $\sigma=G \cap K$. For $q$ lines chosen as random and

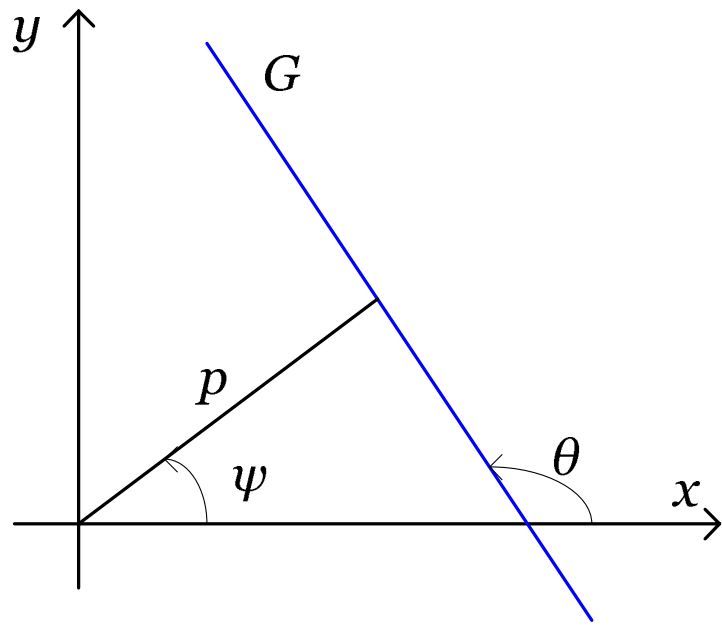

Figure 1. Random Line Model

intersect the bounded convex set $K$, the mean of a sum of all segments of lines interior the convex $K$ is given by $\mathbb{E}_{q}\{s\}=q \mathbb{E}_{K}\{\sigma\}=q \pi F / L \quad$ [20, eq. (4.37)], where $s=\sum_{i} \sigma_{i}$. In the limit case, assume that $K(t)$ expands to the whole plane and $q=q(t) \rightarrow \infty$ in such a way that

$$
\lim _{t \rightarrow \infty} \frac{q(t)}{L(t)}=\frac{1}{2} \tau,
$$

where $\tau$ is positive constant which is the mean value of the number of lines crossing an arbitrary line segment of unit length. The number of lines intersecting $K$ has Poisson distribution with parameter $\frac{1}{2} \tau L$ [21]. The probability that $K$ intersected by exactly $m$ lines is therefore given by

$$
P_{M}(m)=\frac{\left(\frac{1}{2} \tau L\right)^{m}}{m !} e^{-\frac{1}{2} \tau L},
$$

so the mean number of lines intersecting $K$ is $\frac{1}{2} \tau L$. Then this process called homogenous Poisson line process with parameter $\tau$.

Now we model the roads system in urban area as a set of random lines $\left\{G_{i}\left(p_{i}, \psi_{i}\right)\right\}_{i \geq 1}$ which is considered as a homogenous stationary Poisson line process with parameter $\tau$. Therefore, the total length expectation of all segments within the convex set $K$ that lines intersect with $K$ is given by [22]

$$
\mathbb{E}\{s\}=\frac{1}{2} \pi \tau F .
$$

\subsection{Fading Channel Model}

The channel fading in this system is modelled as Nakagami- $m$ fading channel with the distribution and density (in power) are

$$
\begin{aligned}
& F_{\mathrm{f}}(x)=1-\frac{\Gamma_{i c}(m, m x)}{\Gamma(m)}, \\
& f_{\mathrm{f}}(x)=\frac{m^{m} x^{m-1} e^{-m x}}{\Gamma(m)},
\end{aligned}
$$

where $\Gamma_{i c}$ denotes the upper incomplete gamma function. The distribution is a single-parameter version of the Nakagami- $m$ with the mean is always 1 . 


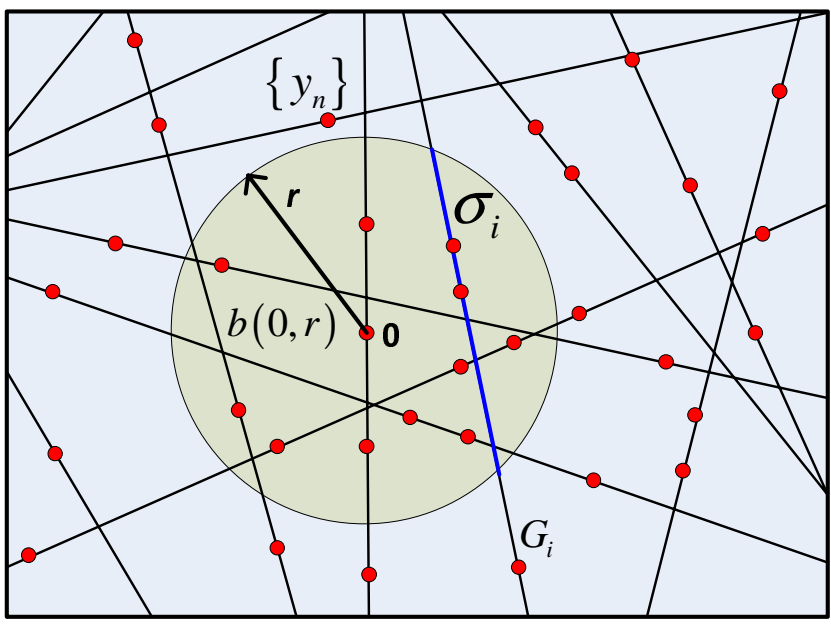

Vehicles spatial distribution - Cox Process

Figure 2. Vehicles Spatial Distribution and COX process

\subsection{Vehicle Locations Model and COX Process}

The location $\left\{y_{n}\right\}$ of vehicles on each random segment $\sigma_{i}=G_{i} \cap K$, an intersection of line $G_{i}$ and convex set $K$, are modelled as linear stationary Poisson point process with linear intensity $\lambda_{l}>0$ (mean number of vehicles per unit length). The number of points $M$ on a segment of length $l$ is a Poisson distributed, $\operatorname{Poi}\left(l \lambda_{l}\right)$, and given $M=m$ points are independent and uniformly distributed on the segment. Here, we consider a convex set $K$ is $b(0, r)$, a disk centered at 0 and radius $r$ with area $F=\pi r^{2}$ and perimeter $L=2 \pi r$. Based on [23] and [24], we suppose that $\sigma_{i}$ is stationary, its distribution is invariant with respect to shifts of the origin $O$, then the process of vehicle locations $\left\{y_{n}\right\}$ on $\mathbb{R}^{2}$ is a Poisson process, as shown in figure 2 , called COX process with mean measure

$$
\begin{aligned}
\Lambda(b(0, r)) & =\Lambda(r)=\lambda_{l} v_{1}\left(\sum_{i=1}^{\infty} G_{i} \cap b(0, r)\right) \\
& =\lambda_{l}\left(\sum_{i=1}^{\infty} \sigma_{i}\right)=\lambda_{l}\left(\sum_{i=1}^{\infty} \frac{\pi F\left(\frac{1}{2} \tau L\right)^{i}}{i !} e^{-\frac{1}{2} \tau L}\right) \\
& =\frac{1}{2} \lambda_{l} \tau \pi F=A r^{2}
\end{aligned}
$$

where $v_{d}(\cdot)$ is $d$-dimensional Lebesgue measure, and $A=\frac{1}{2} \lambda_{l} \tau \pi^{2}$. Thus, probability of $k$ vehicles or nodes in $b(0, r)$ is given by

$$
\begin{aligned}
p_{k}(r) & =\operatorname{Pr}\left\{\lambda_{l} v_{1}\left(\sum_{i=1}^{\infty} G_{i} \cap b(o, r)\right)=k\right\} \\
& =\frac{(\Lambda(r))^{k}}{k !} e^{-\Lambda(r)} .
\end{aligned}
$$

Note that we use the terms nodes and points interchangeably which are standing for locations of vehicles.

\subsection{Distance distribution to the $n$th nearest neighbor}

Let $R_{n}$ be the Euclidean distance from the $n$th nearest neighbor to the origin (arbitrary chosen), and the distribution of $R_{n}$ is given by [25]

$$
f_{R_{n}}(r)=\frac{2}{r} \frac{(\Lambda(r))^{n}}{\Gamma(n)} e^{-\Lambda(r)}=\frac{2}{r} \frac{\left(A r^{2}\right)^{n}}{\Gamma(n)} e^{-A r^{2}},
$$

and the CDF of the distance function

$$
F_{R_{n}}(r)=1-\frac{\Gamma_{i c}\left(n, A r^{2}\right)}{\Gamma(n)} .
$$

The moments of $R_{n}$ are

$$
\begin{aligned}
& \mathbb{E}\left\{R_{n}\right\}=\frac{\Gamma\left(n+\frac{1}{2}\right)}{\sqrt{A} \Gamma(n)}, \\
& \mathbb{E}\left\{R_{n}^{\alpha}\right\}=\frac{\Gamma\left(n+\frac{\alpha}{2}\right)}{A^{\alpha / 2} \Gamma(n)} .
\end{aligned}
$$

\subsection{Point Processes}

In this section, we review the technique of the incorporating fading characteristics into the point process [26] and derive the cumulative distribution function of $n$th nearest neighbor with an effect of path loss and fading. Let the set $\left\{y_{i}\right\}, i \in \mathbb{N}$ consist of the points (vehicles's locations) in $\mathbb{R}^{2}$ of a stationary Poisson point process $\Phi_{y}$ (COX process) with mean measure in (6), be ordered according to their Euclidean distance $\left\|y_{i}-0\right\|$ to the origin 0. Due to the mapping theorem in [23] and [24], we define new one dimensional Poisson point process PPP (generally inhomogenous PPP) $\Phi_{R}=\left\{r_{i} \triangleq\right.$ $\left.\left\|y_{i}-0\right\|\right\}$ such that $0<r_{1}<r_{2}<\ldots$ with mean measure

$$
\Lambda_{R}(0, x) \triangleq \Lambda_{R}(x)=\Lambda(b(o, x))=\frac{1}{2} \lambda_{l} \tau \pi^{2} x^{2}=A x^{2},
$$

and the intensity

$$
\lambda_{R}(x)=\frac{d \Lambda_{R}(x)}{d x}=\lambda_{l} \tau \pi^{2} x=2 A x
$$

Let $\alpha>0$ be the path loss exponent. We define path loss point process before fading $\Phi_{P}=\left\{x_{i} \triangleq r_{i}^{\alpha}\right\}$ which is also a Poisson point process (due to mapping theorem $[23,24])$ with mean measure

$$
\begin{aligned}
\Lambda_{P}(0, x) & \triangleq \Lambda_{P}(x) \\
& =\Lambda_{R}\left(x^{1 / \alpha}\right)=\frac{1}{2} \lambda_{l} \tau \pi^{2} x^{\frac{2}{\alpha}}=A x^{\frac{2}{\alpha}},
\end{aligned}
$$

and the intensity

$$
\lambda_{P}(x)=\frac{d \Lambda_{P}(x)}{d x}=\lambda_{l} \tau \pi^{2} \frac{1}{\alpha} x^{\frac{2-\alpha}{\alpha}}=A \frac{2}{\alpha} x^{\frac{2-\alpha}{\alpha}} .
$$

Let $\left\{f_{1}, f_{2}, \ldots\right\}$ be independent and identically distributed stochastic process with unit mean, $\mathbb{E}\left\{f_{i}\right\}=1$, and $\operatorname{supp} f_{i} \subset \mathbb{R}^{+}$. We define the path loss point process with fading $\Phi_{F}=\left\{\xi_{i} \triangleq \frac{x_{i}}{f_{i}}\right\}$ which is a Poisson point process and $f_{i}$ are i.i.d.

We denote $P_{R x s}$ as the sensibility of receiver according to the modulation scheme and the designed QoS (Quality of Service) of the system, and $P_{R x}$ be a received signal power level, which depends on a channel characteristics and system parameters, i.e., transmitted power, antennas gain. Two nodes with distance $R$ are said to be connected to each other if a received power level 


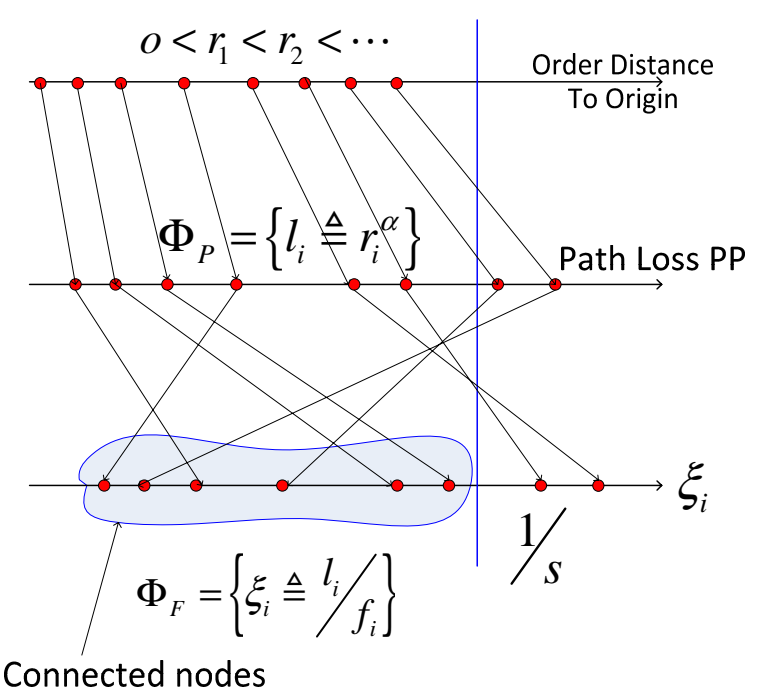

Figure 3. Point Processes for VANET

is greater than the sensibility of receiver, $P_{R x}>P_{R x s}$. Thus, the probability of a successful packet reception is a probability of received power greater than the receiver sensibility. The following equation (16) indicates the model for determining the received signal power with simple path loss calculation

$$
P_{R x}=\frac{P_{T x} D_{T x} D_{R x}}{R^{\alpha}}\left(\frac{c}{f_{c} 4 \pi}\right)^{2},
$$

where $P_{T x}$ is a transmitted power, $D_{T x}$ is a transmission antenna gain, $D_{R x}$ is a receive antenna gain, $c$ is a speed of light, $f_{c}$ is a central frequency. Therefore, two nodes are connected each other when $\frac{P_{T x} D_{T x} D_{R x}}{R^{x}}\left(\frac{c}{f_{c} 4 \pi}\right)^{2}>$ $P_{R x s}$, or equivalent to the path loss is smaller than $R^{\alpha}<\frac{P_{T x} D_{T x} D_{R x}}{P_{R x s}}\left(\frac{c}{f_{c} 4 \pi}\right)^{2}$. For simplifying, we define the path loss threshold, $\frac{1}{s} \triangleq R^{\alpha}$, and $s$ is called minimum path gain threshold for connection. Then we have the process of connected nodes

$$
\hat{\Phi}_{P} \triangleq\left\{x_{i}: \xi_{i}<1 / s\right\}
$$

or

$$
\hat{\Phi}_{F} \triangleq\left\{\xi_{i}: \xi_{i}<1 / s\right\}=\Phi_{F} \cap[0,1 / s) .
$$

The point processes models for the VANETs are shown in Figure 3.

Proposition 1. The CDF and PDF of $x_{i}$ in the the path loss point process before fading $\Phi_{P}$ are given by

$$
\begin{aligned}
& F_{x_{i}}(x)=1-\frac{\Gamma_{i c}\left(i, A x^{2 / \alpha}\right)}{\Gamma(i)}, \\
& f_{x_{i}}(x)=\frac{2}{\alpha x} \frac{\left(A x^{2 / \alpha}\right)^{i}}{\Gamma(i)} e^{\left(-A x^{2 / \alpha}\right)},
\end{aligned}
$$

and the CDF of $\xi_{i}$ in path loss point process with fading $\Phi_{F}$ is expressed as

$$
\begin{aligned}
& F_{\xi_{i}}(x)=\frac{(i-1) !}{\Gamma(i) \Gamma(m)}\{\Gamma(m) \\
& \left.-\sum_{k=0}^{i-1} \frac{\left(A x^{(2 / \alpha)}\right)^{k}}{k !} H_{1,1}^{1,1}\left[A x^{2 / \alpha} \mid \begin{array}{l|l}
\left(1-\frac{2 k}{\alpha}-m, \frac{2}{\alpha}\right) \\
(0,1)
\end{array}\right]\right\} \\
& \quad, i=1,2 \ldots
\end{aligned}
$$

\section{Statistical Characteristics of VANET's CONNECTIVITY}

It is well known that there are various researches of connectivity in MANETs. However, rarely analytical researches of connectivity in VANETs have been carried out. In $[12,27]$ the authors use queueing theory approach for analysis a connectivity in high way scenario of VANETs. Reference [13] uses polytope graph theory for exploiting the impact of dynamic node distribution and delay constraint of a linear network in deterministic channel. In this paper, we apply the point processes including with Nagakami- $m$ fading channel for analyzing VANETs connectivity in two dimensional network under urban area scenario. First, we address statistical properties for one hop connectivity such as successful probability, mean distance, mean number of connected node, and node density requirement for subarea has high connectivity probability. The outage probability, maximum number of hops, and maximal broadcast percolation distance of the connectivity between the origin and the $n$th nearest neighbor are also computed.

\subsection{One-Hop Connectivity Property}

Proposition 2. We consider a node with path loss $x$, the probability of this node to be connected successfully to the origin in the Nakagami-m fading environment is given by

$$
P_{\text {succ }}(x)=1-F_{\mathrm{f}}(s x),
$$

with the mean number of connected nodes

$$
\mathbb{E}\left\{\hat{N}_{m}\right\}=\frac{A}{(m s)^{\frac{2}{\alpha}}} \frac{\Gamma\left(m+\frac{2}{\alpha}\right)}{\Gamma(m)},
$$

probability of a node to be isolated

$$
\operatorname{Pr}\{i s o\}=\exp \left\{-\frac{A}{(m s)^{\frac{2}{\alpha}}} \frac{\Gamma\left(m+\frac{2}{\alpha}\right)}{\Gamma(m)}\right\},
$$

and the mean distance of a connected node

$$
\mathbb{E}\left(x^{1 / \alpha}\right)=\frac{2}{3} \frac{1}{(m s)^{\frac{1}{\alpha}}} \frac{\Gamma\left(\frac{3}{\alpha}+m\right)}{\Gamma\left(\frac{2}{\alpha}+m\right)} .
$$

Proposition 3. We consider a subarea $\mathbf{B}$ of the infinity system plane, i.e., a circular area with radius $\rho$. The network formed by nodes in $\mathbf{B}$ is said to be connected if and only if there is exist at least a path between any pair of nodes in B. For given channel statistical characteristics, $\alpha$ and $m$, path loss threshold, $1 / s$, roads system property, $\tau$. The lower bound requirement density of cars, $\lambda_{l}$, that the 
network connectivity in this subarea with high probability, (i.e. $p_{0}=99 \%$ ) is given by

$$
\begin{aligned}
\lambda_{l}\left(\operatorname{Pr}\{\operatorname{con}\}=p_{0}\right) & \geq-\frac{2}{\tau \pi^{2}} \frac{(m s)^{2 / \alpha} \Gamma(m)}{\Gamma(m+2 / \alpha)} \\
& \times W_{-1}\left(\frac{\ln \left(p_{0}\right)}{\rho^{2}} \frac{\Gamma(m+2 / \alpha)}{(m s)^{2 / \alpha} \Gamma(m)}\right),
\end{aligned}
$$

where $W_{-1}(\cdot)$ denotes the real-valued, lower branch of the LambertW function ${ }^{1}$, and $\ln (\cdot)$ is the natural logarithm.

\subsection{The $n$th Nearest Neighbor Connectivity Property}

Now we consider the outage probability of the $n$th nearest neighbor to the origin which is defined as the probability of their path loss with fading is greater than the path loss threshold, $1 / \mathrm{s}$. We also consider the maximal number of hops based on the given outage constraint. We assume that the source node knows the direction towards the $n$th nearest neighbor.

Proposition 4. The outage probability of the $n$th nearest neighbor to the origin is given by

$$
P_{\text {out }}\left(n, \frac{1}{s}\right)=1-F_{\xi_{n}}\left(\frac{1}{s}\right),
$$

where $F_{\xi_{n}}(\cdot)$ from $(21)$.

Proof: Based on the definition of outage probability, probability of two nodes can not communicate each other, and the definition of connected node process (18), we have $P_{\text {out }}\left(n, \frac{1}{s}\right)=\operatorname{Pr}\left\{\xi_{n} \geq \frac{1}{s}\right\}=1-\operatorname{Pr}\left\{\xi_{n}<\frac{1}{s}\right\}$, then the proof is complete.

Proposition 5. The maximal number of hops for the $n$th nearest neighbor with a given outage probability constraint $\epsilon$ is

$$
M_{n, \epsilon}=\left\lfloor\frac{\ln (1-\epsilon)}{\ln \left(1-F_{\xi_{n}}\left(\frac{1}{s}\right)\right)}\right\rfloor,
$$

where $\lfloor z\rfloor$ is the greatest integer number that is less than $z$.

Remark 1. In the VANETs, information needs to be disseminated through out a network. So the ability of a message broadcast to percolate through a network according to a radio transmission power and station density is also concerned. Based on the maximum number of hops, we can further determine how far in average a message can be traversed while maintaining network constraints of outage probability $\epsilon$ as follows:

$$
L_{\max , n}=\sum_{i=1}^{M_{n, \epsilon}} \mathbb{E}\left\{R_{i, i+n}\right\},
$$

where $\mathbb{E}\left\{R_{i, i+n}\right\}$ is from (10) and $M_{n, \epsilon}$ is from (28).

\footnotetext{
${ }^{1}$ The definition of the LambertW function is that it satisfies $w(z) e^{w(z)}=z$. If $z$ is a real value, $w(z) \geq-1$ we denote $w_{0}(z)$, called principal branch or higher branch. when $w(z)<-1$, we denote $w_{-1}(z)$, called non-principal or lower branch
}

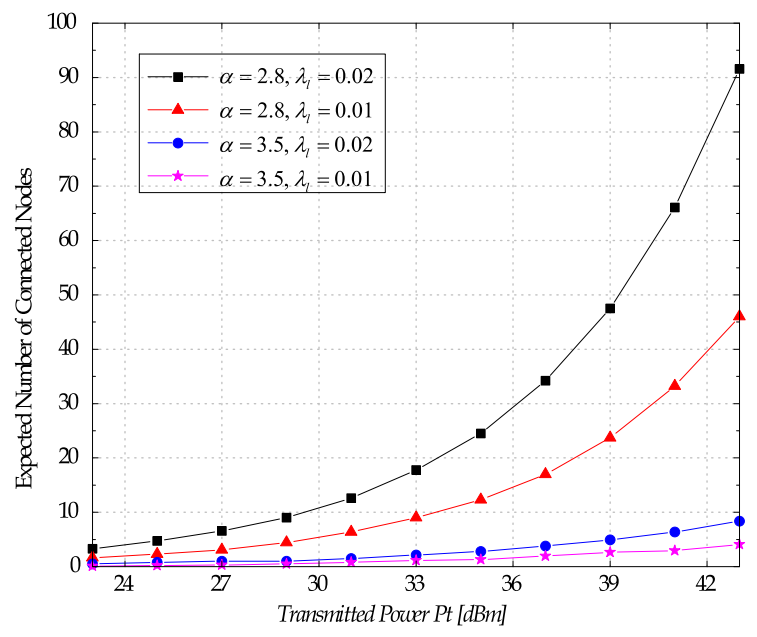

Figure 4. Mean Number of Connected Node to The Origin with Different Vehicle Density and Path Loss Exponent with $\tau=0.0072$.

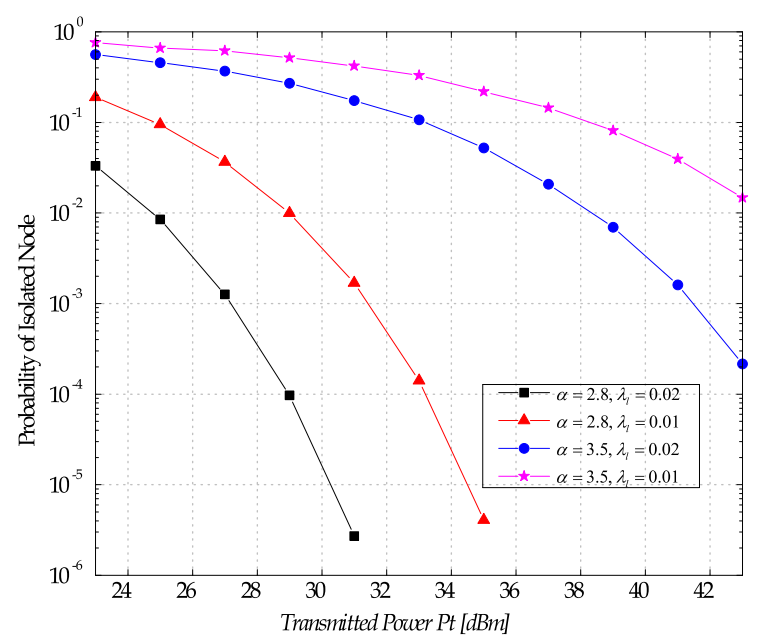

Figure 5. Probability of An Isolated Node with Different Vehicle Density and Path Loss Exponent with $\tau=0.0072$.

\section{Numerical Examples}

In this section, we give some numerical examples of the statistical properties of VANETs connectivity with road system parameter $\tau=0.0072$, i.e., 15462 intersections over $2146 \mathrm{~km}$, number of intersections per meter, according to road data in [28]. The transmitted power, frequency, antenna gains, and path loss exponent are chosen as parameters of DSRC system. For example, transmitted power ranges from $23 \mathrm{dBm}$ to $43 \mathrm{dBm}$, frequency $f_{c}=5.9 \mathrm{GHz}$, transmit and receive antenna gains are 1, path loss exponent is from 2.8 to 5.9. The following figures show the results of the analysis presented above.

\subsection{One-Hop Connectivity Property}

Figure 4 and figure 5 show the mean number of connected nodes to the origin and probability of an isolated node versus transmitted power with different density of vehicle on a road and path loss exponent, respectively. In these figures, we set $\alpha$ to be 2.8 and $3.5, \lambda_{l}$ to be 0.02 and 0.01 . The mean number of connected nodes as a 


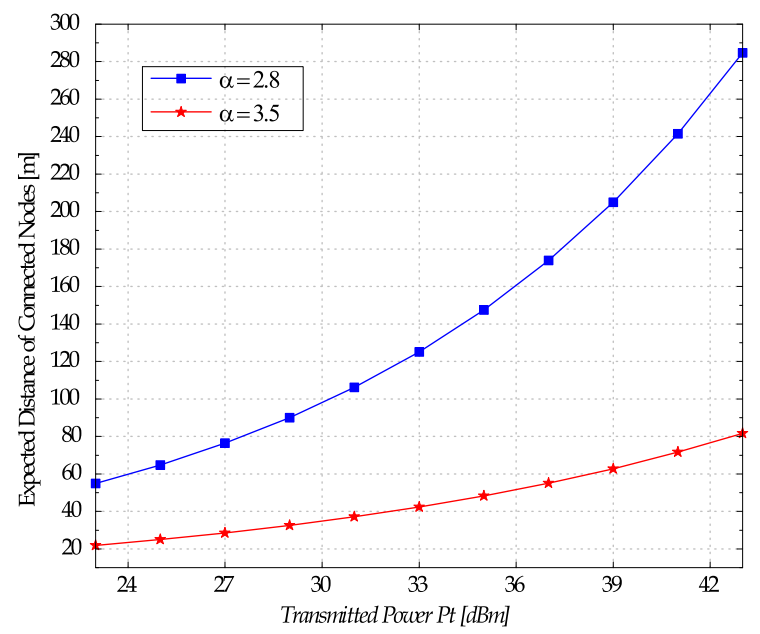

Figure 6. Mean Distance of Connected Node to The Origin with Different Path Loss Exponent.

function of transmitted power is shown in figure 4. It is clear that the expected number of connected nodes is changed much according to the varying of node density and transmitted power as $\alpha$ is small. Otherwise, the number of connected nodes do not increase much for large $\alpha$. Figure 5 shows the probability of a isolated node. For small path loss exponent and large intensity of vehicles, the probability of isolated node reduced quickly as increasing transmitted power. However, this reduction becomes smaller as $\alpha$ becomes larger.

Figure 6 shows the mean distance of a connected node to the origin versus transmitted power. For small path loss exponent, mean distance increases much when transmitted power goes up while it changes slowly according to transmitted power for the large path loss exponent case.

Due to the requirement of consistent connection for safety application in VANETs, the network have to always maintain a full connection status. Here we consider a lower bound of vehicle density requirement for full connection of subarea with radius $\rho$. Figure 7 shows the lower bound density of vehicle for probability of 0.99 of connectivity versus to transmitted power with different areas and path loss exponent. The computed values of the critical vehicle density are practical relevance for designing multihop wireless networks, i.e., we can add more road side units (RSUs) for some areas with statistically low density of vehicle to obtain high probability of connection.

\subsection{The $n$th Nearest Neighbor Connectivity Property}

In figure 8 and figure 9, we plot outage probability of a $n$th nearest neighbor hop versus to transmitted power with different routing strategies and path loss exponents. It is clearly seen that outage probability of $n$th nearest neighbor hop increases with transmitted power and reduced with the number $n$, which are shown in figure 8 . The impact of path loss and node density on the outage are shown in figure 9. In figure 10, maximum number of hops for a given multihop outage probability are addressed for different routing

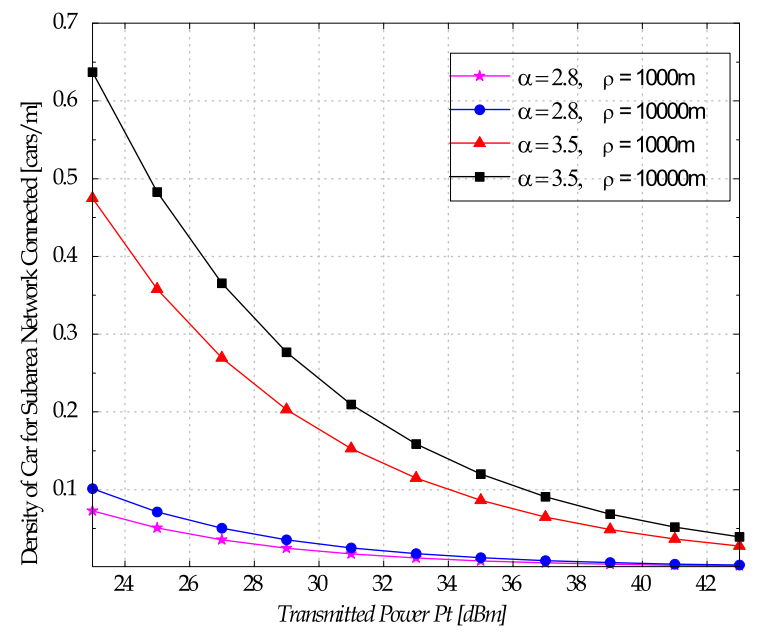

Figure 7. Lower Bound Density of Vehicles for Subarea Connectivity with High Probability vs Transmitted Power with Different Path Loss Exponents and Areas with $\tau=0.0072$ and $p_{0}=0.99$.

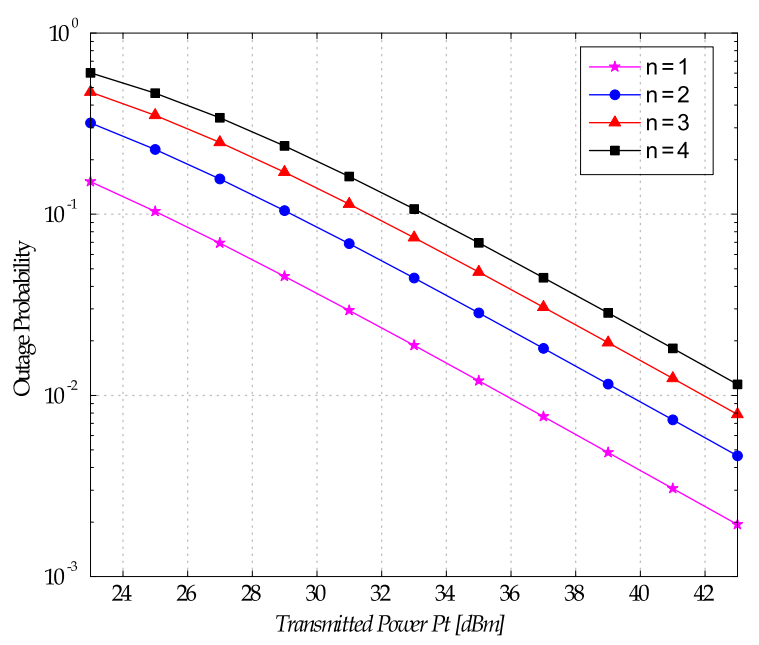

Figure 8. Outage probability of $n$th nearest neighbor connection vs transmitted power with $\tau=0.0072, \alpha=2.8$, and $\lambda_{l}=0.02$.

strategies ( $n$th nearest neighbor routing strategy) with a given outage, $\epsilon$. It is also a function of transmitted power, node density, path loss and routing policies. The maximal distance of broadcast percolation is a function of routing strategy, transmitted power, vehicle density, path loss exponent and requirement outage probability. The dependence on those parameters are shown in the figure 11 and figure 12. For a given quality of service and system parameters, we can estimate how far a message can be percolated in a network.

\section{Conclusions}

In this paper, we propose a stochastic geometry model of a road system for VANETs connectivity analysis in urban area environment. By using this proposed model, a probability of a node to be connected to arbitrary origin and mean number of connected node for a given system parameters can be estimated easily. We can find the requirement of node density for a subarea connected with high probability. For different routing 


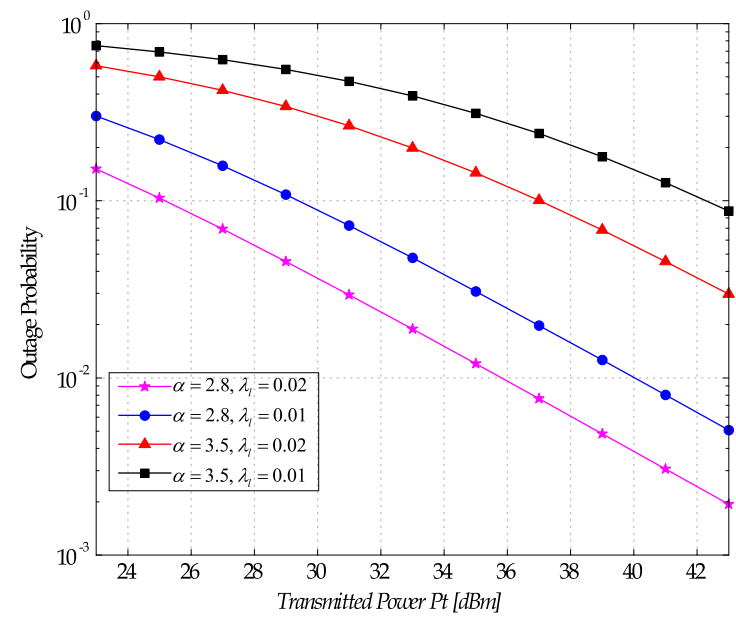

Figure 9. Outage probability of $n$th nearest neighbor connection vs transmitted power with different Path loss and Node Density with $\tau=0.0072$ and $n=1$

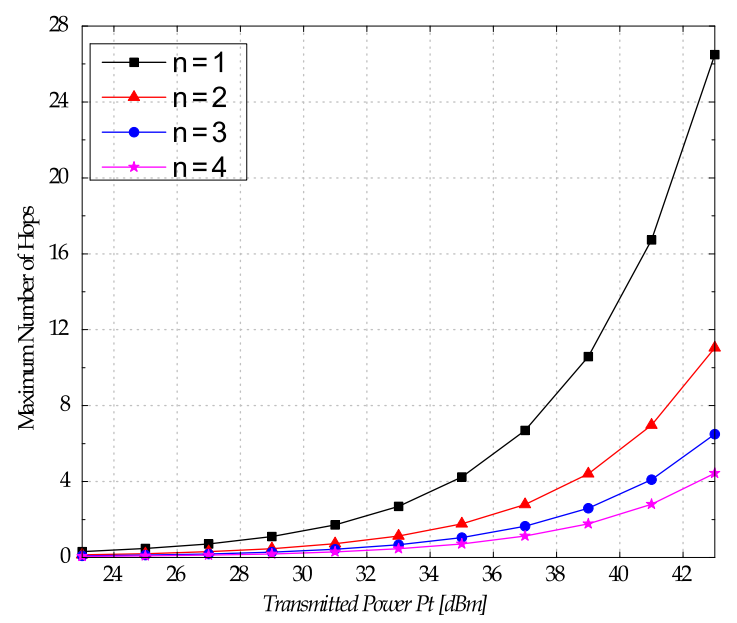

Figure 10. Maximum number of hops for $n$th nearest neighbor routing vs Transmitted Power with $\epsilon=0.05, \tau=0.0072, \alpha=2.8$, and $\lambda_{l}=0.02$.

strategies, we can calculate the outage probability and maximum number of hops as well as how far a message can be broadcast in a network. Therefore, the proposed model can be used as a powerful adaptive tool for design, planning and modification for improving a network design according to rapid changes and complex requirements.

\section{ApPENDIX}

\section{A.1 Proof of Proposition 1}

We have the CDF of $x_{i}$ as $F_{x_{i}}(x)=\operatorname{Pr}\left\{x_{i} \leq x\right\}=$ $\operatorname{Pr}\left\{r_{i} \leq x^{1 / \alpha}\right\}=F_{R_{i}}\left(x^{1 / \alpha}\right)$. From (9) we get the equation (19). After differentiating this equation we have the PDF function of $x_{i}$ as in (20). Now we prove the CDF of $\xi_{i}$, from the definition of path loss point processes with

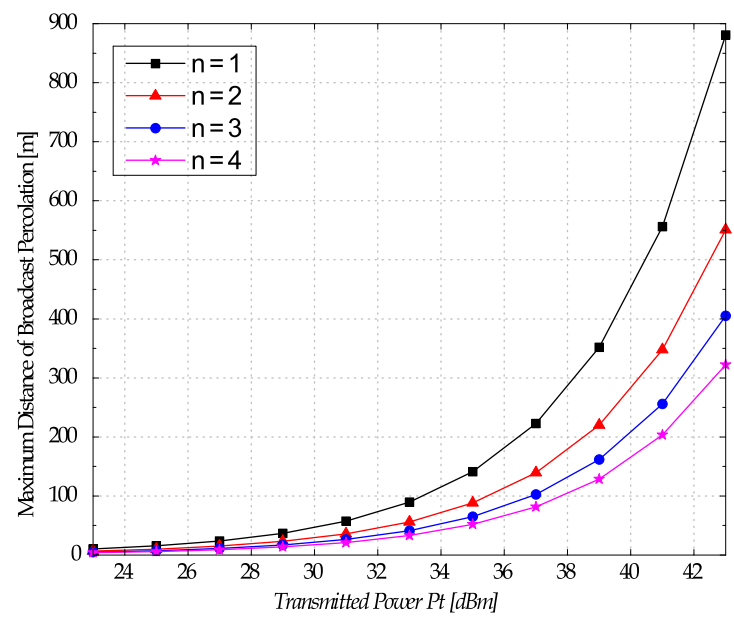

Figure 11. Maximum Distance of Broadcast Percolation for $n$th nearest neighbor routing vs Transmitted Power with $\epsilon=0.05, \lambda_{l}=0.02$, $\tau=0.0072$, and $\alpha=2.8$.

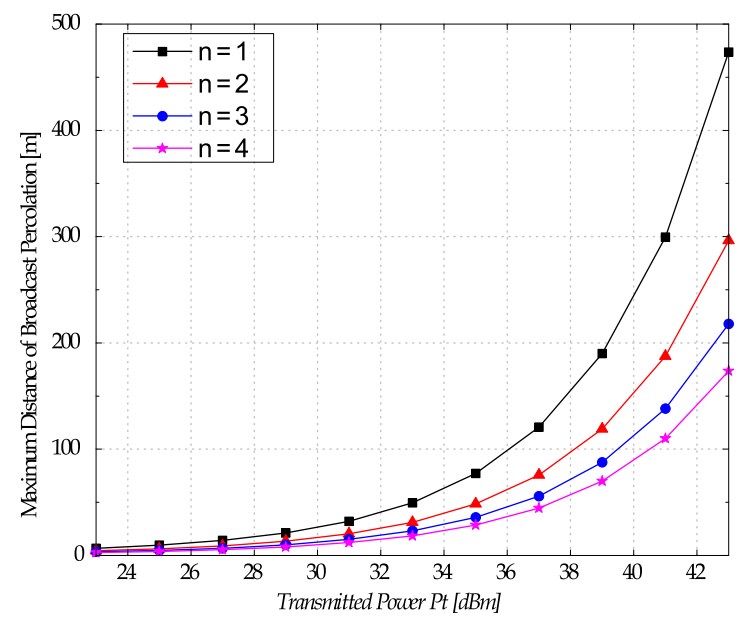

Figure 12. Maximum Distance of Broadcast Percolation for $n$th nearest neighbor routing vs Transmitted Power with $\epsilon=0.05, \lambda_{l}=0.01$, $\tau=0.0072$, and $\alpha=2.8$.

fading, we have

$$
\begin{aligned}
F_{\xi_{i}}(x) & =\operatorname{Pr}\left\{\xi_{i} \leq x\right\}=\operatorname{Pr}\left\{\frac{x_{i}}{f_{i}} \leq x\right\}=\operatorname{Pr}\left\{f_{i} \geq \frac{x_{i}}{x}\right\} \\
= & \frac{(i-1) !}{\Gamma(i) \Gamma(m)}\{\Gamma(m) \\
- & \left.\sum_{k=0}^{i-1} \frac{\left(A x^{(2 / \alpha)}\right)^{k}}{k !} \int_{0}^{\infty} t^{\left(\frac{2 k}{\alpha}+m-1\right)} e^{-t} e^{-A x^{2 / \alpha} t^{2 / \alpha}} d t\right\} \\
, i=1,2 \ldots & \text { (A. } 1)
\end{aligned}
$$

We express $e^{-A x^{2 / \alpha} t^{2 / \alpha}}$ in terms of Meijers Gfunction [29, eq. (8.4.3.1)] as follows

$$
e^{-A x^{2 / \alpha} t^{2 / \alpha}}=G_{0,1}^{1,0}\left(\begin{array}{l|l}
A x^{2 / \alpha} t^{2 / \alpha} & - \\
0
\end{array}\right),
$$

then using [30, eq. (1.7.1)] and (A.2), we have

$$
e^{-A x^{2 / \alpha} t^{2 / \alpha}}=H_{0,1}^{1,0}\left[\begin{array}{l|l}
A x^{2 / \alpha} t^{2 / \alpha} & (-) \\
(0,1)
\end{array}\right] .
$$

By substituting (A.3) in (A.1) and using [29, eq. (2.25.2.3)], we obtain (21) and complete the proof. 


\section{A.2 Proof of proposition 2}

We define a node to be connected to the origin if the path loss with fading is lesser than the path loss threshold which defined in (18). So, the probability of a node to be connected successfully is

$$
\begin{aligned}
P_{\text {succ }}(x) & =\operatorname{Pr}\left\{\xi<\frac{1}{s}\right\}=\operatorname{Pr}\left\{\frac{x}{f}<\frac{1}{s}\right\} \\
& =\operatorname{Pr}\{s x<f\}=1-F_{\mathrm{f}}(s x) .
\end{aligned}
$$

Thus, the connected nodes process $\hat{\Phi}_{P}$ is a thinning Poisson process [24] with intensity $\hat{\lambda}(x)=$ $\lambda(x)\left(1-F_{\mathrm{f}}(s x)\right)$, then the mean number of connected nodes in the system is given by

$$
\begin{aligned}
\mathbb{E}\left\{\hat{N}_{m}\right\} & =\int_{0}^{\infty} \hat{\lambda}(x) d x \\
& =\int_{0}^{\infty} A \frac{2}{\alpha} x\left(\frac{2}{\alpha}-1\right) \frac{\int_{m s x}^{\infty} t^{m-1} e^{-t} d t}{\Gamma(m)} d x .
\end{aligned}
$$

After integrating the (A.5), we get the (23). If there is no fading channel

$$
\begin{aligned}
\mathbb{E}\left\{\hat{N}_{\infty}\right\} & =\lim _{m \rightarrow \infty} \mathbb{E}\left\{\hat{N}_{m}\right\}=\Lambda_{P}\left(\frac{1}{s}\right) \\
& =\int_{0}^{1 / s} \lambda_{P}(x) d x=A s^{-\frac{2}{\alpha}} .
\end{aligned}
$$

Since the point process of the connected nodes is thinning Poisson point process, the probability of an isolated node is $\operatorname{Pr}\{$ iso $\}=\operatorname{Pr}\left\{\hat{N}_{m}=0\right\}=\exp \left(-\mathbb{E}\left(\hat{N}_{m}\right)\right)$. Substituting $-\mathbb{E}\left(\hat{N}_{m}\right)$ from (23) in this equation we get (24). Finally, we have distribution of a random connected node $f_{\hat{x}}(x)=\frac{\hat{\lambda}(x)}{\mathbb{E}\left(\hat{N}_{m}\right)}$, so the mean distance of a connected nodes is $\mathbb{E}\left(x^{1 / \alpha}\right)=\int_{0}^{\infty} x^{1 / \alpha} f_{\hat{x}}(x) d x$, then we get (25) and the proof is complete.

\section{A.3 Proof of Proposition 3}

We know that it is not sufficient condition for network of given subarea to be connected if there is no isolated node. Thus, probability of non of nodes in $\mathbf{B}$ is isolated (denotes $\operatorname{Pr}\{n o$ iso $\}$ is an upper bound for the probability that all nodes in $\mathbf{B}$ are connected. Consequently, node density required to achieve probability of no isolated node at certain value, $p_{0}$, is a lower bound for node density required to achieve subnetwork connected with the same probability $p_{0}$,

$$
\lambda_{l}\left(\operatorname{Pr}\{\text { con }\}=p_{0}\right) \geq \lambda_{l}\left(\operatorname{Pr}\{\text { no iso }\}=p_{0}\right) .
$$

Now we calculate the right handed side of (A.7). The isolation of different nodes can be considered to be almost independent statistical events. Hence, the conditional probability is

$$
\operatorname{Pr}\{\text { no iso } \mid N=k\}=(1-\operatorname{Pr}\{i s o\})^{k} .
$$

Assuming that the number of nodes in $\mathbf{B}$ is known, i.e., $k=N$. Substituting the $\operatorname{Pr}\{$ iso $\}$ from (24) in (A.8), so the unconditional no isolated node becomes

$$
\begin{aligned}
& \operatorname{Pr}\{\text { no iso }\}=\sum_{k=0}^{\infty} \operatorname{Pr}(\{\text { no iso } \mid N=k)\} P(N=k) \\
& =\exp \left(-1 / 2 \lambda_{l} \tau \pi^{2} \rho^{2} \exp \left(\frac{-1 / 2 \lambda_{l} \tau \pi^{2}}{(m s)^{2 / \alpha}} \frac{\Gamma(m+2 / \alpha)}{\Gamma(m)}\right)\right) .
\end{aligned}
$$

Let $\operatorname{Pr}\{$ no iso $\}=p_{0}$, we solve the equation (A.9) for $\lambda_{l}$ leads to the result

$$
\begin{aligned}
\lambda_{l}\left(\operatorname{Pr}\{\text { no iso }\}=p_{0}\right) & =-\frac{2}{\tau \pi^{2}} \frac{(m s)^{2 / \alpha} \Gamma(m)}{\Gamma(m+2 / \alpha)} \\
& \times W_{-1}\left(\frac{\ln \left(p_{0}\right)}{\rho^{2}} \frac{\Gamma(m+2 / \alpha)}{(m s)^{2 / \alpha} \Gamma(m)}\right) .
\end{aligned}
$$

Combine equation (A.7) and equation (A.10) we get the lower bound of car density (26), and the proof is complete.

\section{A.4 Proof of Proposition 5}

For the $n$th nearest neighbor routing strategy with a given outage constraint $\epsilon$, we find the maximal number of hops, $M$. The signal has travelled from node $S_{1}$ to node $S_{M}$ via $M$ hops. We assume that there are $M-1$ nodes working as relay nodes. The relay will communicate to the $n$th nearest neighbor (as the next relay node) and so on until reaching the last node $S_{M}$.

The outage probability of the path from node $S_{1}$ to node $S_{M}$ is a probability of at least one hop outage,

$$
\begin{aligned}
& P_{\text {out }}\left(n, M, \frac{1}{s}\right)= \\
& \operatorname{Pr}\left\{\min \left\{\xi_{1, n+1}, \xi_{n+1,2 n+1}, \ldots, \xi_{M n+1,(M+1) n+1}\right\} \geq \frac{1}{s}\right\} \\
& =1-\prod_{i=1}^{M}\left(1-\operatorname{Pr}\left\{\xi_{i, i+n}<\frac{1}{s}\right\}\right), i=1,2 \ldots
\end{aligned}
$$

Assuming that $\xi_{i, i+n}$ are independent and identically distributed (i.i.d), then we have

$$
P_{\text {out }}\left(n, M, \frac{1}{s}\right)=1-\left(1-F_{\xi_{n}}\left(\frac{1}{s}\right)\right)^{M} \text {. }
$$

This outage probability must lesser than the given outage probability constraint, $P_{\text {out }}\left(n, M, \frac{1}{s}\right) \leq \epsilon$, by solving the inequality we get the result (28), the proof is complete.

\section{REFERENCES}

[1] S. Olariu and M. C. Weigle, Vehicular Networks: From Theory to Practice, 1st ed. 6000 Broken Sound Parkway, NW: CRC Press, 2009.

[2] M. M. Artimy, W. Robertson, and W. J. Phillips, "Vehicle traffice microsimulator for Ad Hoc networks research," in Proc. of International Workshop on Wireless Ad Hoc Networks (IWWAN) 2004, Oulu, Finland, May 2004, pp. $1-5$.

[3] M. kafsi, P. Papadimitratos, O. Dousse, T. Alpcan, and J. P. Hubaux, "VANET connectivity analysis," in Proc. 
IEEE Workshop on Automotive Networking and Applications (Autonet),2008, New Orleans, LA, USA, Dec. 2008.

[4] G. H. Mohimani, F. Ashtiani, A. Javanmard, and M. Hamdi, "Mobility modelling, spatial traffice distribution, and probability of connectivity for sparse and dense vehicular Ad Hoc networks," IEEE Transactions on Vehicular Technology, vol. 58, no. 4, pp. 1998-2007, May 2009.

[5] A. Torok, D. Jozsef, and B. Sonkoly, "A hybrid simulation framework for modeling and analysis of vehicular Ad Hoc networks," in Proc. IEEE Vehicular Technology Conference (VTC Spring), Budapest, Hungary, May 2011, pp. $1-5$.

[6] D. S. Gaikwad and M. Zaveri, "A novel mobility model for realistic behavior in vehicular Ad Hoc network," in Proc. IEEE International Conference on Computer and Information Technology (CIT), Pafos, Cyprus, 2011, pp. 597-602.

[7] E. Giordano, R. Frank, G. Pau, and M. Gerla, "CORNER: A radio propagation model for VANETs in urban scenarios," Proceedings of the IEEE, vol. 99, no. 7, pp. 1280-1294, 2011.

[8] A. Cardote, F. Neves, S. Sargento, and P. Steenkiste, "A statistical channel model for realistic simulation in VANET," in Proc. IEEE Vehicular Networking Conference (VNC), Seoul, Republic of Korea, Nov. 2012, pp. 48-55.

[9] Y. C. Cheng and T. Robertazzi, "Critical connectivity phenomena in multihop radio models," IEEE Transactions on Communications, vol. 37, no. 7, pp. 770-777, 1989.

[10] O. Dousse, P. Thiran, and M. Hasler, "Connectivity in ad-hoc and hybrid networks," in Proc. of the 21th Annual Joint Conference of the IEEE Computer and Communications Societies,INFOCOM02, NY, USA, Dec. 2002, pp. 10791088.

[11] W. Jin and W. W. Recker, "Instantaneous information propagation in a traffic stream through inter-vehicle communication," Transp. Res,", Part B: Methodol., vol. 40, no. 3, pp. 230-250, Mar. 2006.

[12] D. Miorandi and E. Altman, "Connectivity in onedimensional Ad Hoc networks: A queueing theoretical approach," Springer Wireless Nerworks Journal, vol. 12, no. 6 , pp. 573-587, Oct. 2006

[13] J. Wu, "Connectivity of mobile linear networks with dynamic node population and delay constraint," IEEE Journal on Selected Areas in Communications, vol. 27, no. 7, pp. 2118-2125, Sep. 2009.

[14] P. Gupta and P. R. Kumar, "Critical power for asymptotic connectivity in wireless networks," in Proc. 37th IEEE Conference on Decision and Control, Tampa, FL-USA, Dec. 1998, pp. 1106-1109.

[15] P. Santi, "The critical transmitting range for connectivity in sparse wireless Ad Hoc networks," IEEE Transactions on Mobile Computing, vol. 2, no. 1, pp. 25-39, Jan. 2003.

[16] _ " "The critical transmitting range for connectivity in mobile Ad Hoc networks," IEEE Transactions on Mobile Computing, vol. 4, no. 3, pp. 210-317, 2005.

[17] C. Bettstetter and C. Hartmann, "Connectivity of wireless multihop networks in shadow fading environment," Springer Wireless Nerworks Journal, vol. 11, no. 5, pp. 571579, Sep. 2005.

[18] R. Hekmat and P. . V. Mieghem, "Connectivity in wireless Ad Hoc networks with a log-normal radio model," Mobile Networks and Applications, vol. 11, no. 3, pp. 351360, 2006.

[19] F. Voss, C. Gloaguen, F. Fleischer, and V. Schmidt, “Distributional properties of euclidean distances in wireless networks involving road systems," IEEE Journal on Selected Areas in Communications, vol. 27, no. 7, pp. 10471055, Sep. 2009.

[20] L. A. Santaló, Introduction to Integral Geometry, 2nd ed. Canada: Addison Wesley Pub. Company, 1976.

[21] L. A. Santaló and I. Yanez, "Averages for polygons formed by random lines in euclidean and hyperbolic planes," J. Appl. Prob., vol. 9, pp. 140-157, 1972.

[22] P. Parker and R. Cowan, "Some properties of line segment processes," J. Appl. Prob., vol. 13, pp. 96-107, 1976.

[23] J. F. C. Kingman, Poisson Processes, 1st ed. Oxford NewYork: Oxford Science Publications, 1993.

[24] D. Stoyan, W. S. Kendall, and J. Mecke, Stochastic Geometry and its Applications, 2nd ed. United Kingdom: John Wiley and Son, 1995.

[25] M. Haenggi, "On distances in uniformly random networks," IEEE Transactions on Information Theory, vol. 51, no. 10, pp. 3584-3586, Oct. 2005.

[26] — " "A geometric interpretation of fading in wireless networks: Theory and applications," IEEE Transactions on Information Theory, vol. 54, no. 12, pp. 5500-5510, Dec. 2008.

[27] S. Yousefi, E. Altman, R. El-Azouzi, and M. Fathy, “Analytical model for connectivity in vehiclular Ad Hoc networks," IEEE Transactions on Vehicular Technology, vol. 57, no. 6, pp. 3341-3356, Nov. 2008.

[28] C. Gloaguen, F. Voss, and V. Schmidt, "Parametric distance distributions for fixed access network analysis and planning," in Proc. of the 21th International Teletraffic Congress, Paris, Franch, Sep. 2009.

[29] A. P. Prudnikov, Y. A. Brychkov, and O. I. Marichev, Integrals and Series. New York: Gordon and Breach Science, 1990, vol. 3

[30] A. M. Mathai and R. K. Saxena, The H-function with Applications in Statistics and Other Disciplines. New York: Wiley, 1978.

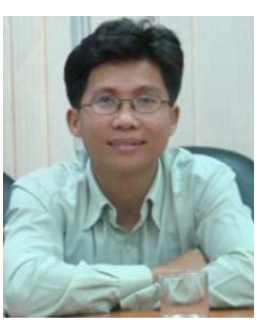

Duong Hien Thuan graduated the Bachelor Engineer Degree in telecommunications from Ho Chi Minh University of Technology, Ho Chi Minh City, Vietnam and Master Engineer in Telecommunications from Asian Institute of Technology (AIT), Thailand, in 1999 and 2002, respectively. He was lecturer at Posts and Telecommunication Institute of Technology (PTIT) in Ho Chi Minh from 1999 to 2009. Currently, he is lecturer at Sai Gon University (SGU). His research interests include coding theory, mobile communication, vehicular ad-hoc networks, multicarrier and multimedia communication systems.

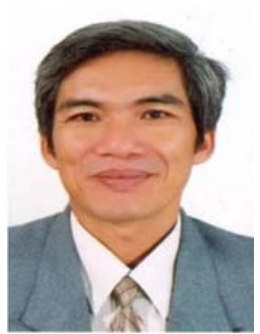

Ho Van Cuu was born in 1964 in Quang Ngai City, Vietnam. He graduated the Bachelor Engineer Degree in telecommunications from Ho Chi Minh City University of Technology, and Master degree in electronic telecommunications from Ha Noi University of Technology in 1987 and 1997, respectively. He received $\mathrm{Ph} . \mathrm{D}$ degree in telecommunications from Post and Telecommunications Institute Technology PTIT in 2007. Currently, he is Head of department of Electronics and Telecommunications of Sai Gon University (SGU). His research interests include mobile communications, digital multiplexer and Radio communication networks.

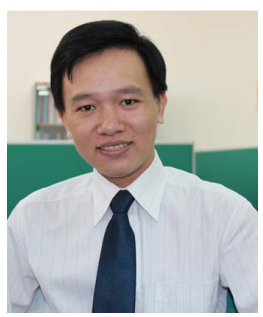

Hung Ngoc Do has been a researcher at school of Electrical Engineering, the International University, Vietnam National University - Ho Chi Minh city (VNU-HCM) Vietnam since 2007. He studied electronics and telecommunications at Ho Chi Minh city University of Technology, Vietnam, where he received Bachelor of Engineering and Master of Engineering in 2002 and 2004, respectively. His main research interests are Cooperative communications, MIMO and OFDM techniques, VANETs, Information theory and Network Coding. 